\title{
Assessment of Attentional Workload while Driving by Eye-fixation-related Potentials
}

\author{
Yuji TAKEDA*, Noritoshi YOSHITSUGU**, Kazuya ITOH** and Nobuhiro KANAMORI* \\ * National Institute of Advanced Industrial Science and Technology, 1-1-1 Higashi, Tsukuba, Ibaraki 305-8566, Japan \\ ** Nissan Motor Co., LTD., 560-2 Okatsu Furuku, Atsugi, Kanagawa 243-0192, Japan
}

\begin{abstract}
How do drivers cope with the attentional workload of in-vehicle information technology? In the present study, we propose a new psychophysiological measure for assessing drivers' attention: eye-fixation-related potential (EFRP). EFRP is a kind of eventrelated brain potential measurable at the eye-movement situation that reflects how closely observers examine visual information at the eye-fixated position. In the experiment, the effects of verbal working memory load and spatial working memory load during simulated driving were examined by measuring the number of saccadic eye-movements and EFRP as the indices of drivers' attention. The results showed that the spatial working memory load affected both the number of saccadic eye-movements and the amplitude of the P100 component of EFRP, whereas the verbal working memory load affected only the number of saccadic eye-movements. This implies that drivers can perform time-sharing processing between driving and the verbal working memory task, but the decline of accuracy of visual processing during driving is inescapable when the spatial working memory load is given. The present study suggests that EFRP can provide a new index of drivers' attention, other than saccadic eye-movements.
\end{abstract}

Keywords: Workload, Attention, Driving, EEG, Eye-fixation-related potential

\section{INTRODUCTION}

In the last two decades, in-vehicle information technology, such as mobile phones or navigation systems, has made spectacular advances. While these devices enhance convenience during driving, they also increase the mental workload of drivers. Indeed, many studies have reported that using an in-vehicle device impaired drivers' attention [1], and lack of attention is one of the main causes of traffic accidents [2]. A valid and reliable assessment of drivers' workload while using in-vehicle devices is necessary for reasons of convenience and safety.

In previous studies investigating the mental workload during driving or simulated driving, the magnitude of the workload has been assessed by reaction times for driverelevant events (e.g., braking) [3] or drive-irrelevant events (e.g., the onset of LEDs on the windshield or a simulated display) [4], by questionnaires [5], by measuring autonomic responses (e.g., heart rate variability) [6], and by measuring eye-movements [7, 8]. However, the measures used in the previous studies are not sufficient to assess drivers' attention in natural driving environments. For example, it is difficult to measure reaction times for driverelevant events during actual driving, because experimenters could not control the events during the actual driving task. Moreover, measuring reaction times for drive-irrelevant events may interfere with natural driving behavior. Questionnaires and autonomic responses cannot provide a direct index of drivers' visual processes. Although eye-movements are measurable in natural driving environments and reflect where the drivers attend to, how closely they examined the information at the eye-fixated location is unmeasurable. The goal of the present study is to propose a new psychophysiological measure for assessing how closely drivers examine the visual information in both simulated and actual driving environments.

An electroencephalogram (EEG) can provide a direct index of cognitive processes, because every cognitive process is associated with activation in the brain. Event related brain potentials (ERPs), which can be obtained by averaging EEG signals time-locked to specific events, reflect many cognitive processes, such as perception, attention, memory, and action. In ergonomic studies, auditory ERP has been used as an index of mental workload during visual tasks $[9,10]$. To measure driver workload in a natural driving environment, events yielding ERPs should be embedded in the driving itself. However, the presentation of task-irrelevant auditory stimulus is necessary for measuring the auditory ERP, which may interfere with the primary task (i.e., driving). Thus, the auditory ERP is not suitable for assessing workload in a natural driving environment. One of the drive-relevant events yielding ERPs is saccadic eye-movements. The ERP is measurable by time-locking to fixation pauses of voluntary saccadic eye-movements [11], referred to as the eye-fixation-related potentials (EFRPs). It has been reported that the P100 component of EFRP varies with 
visual attention $[12,13]$. The P100 component of EFRP can be elicited from the occipital region (i.e., visual cortex) [14], and it reflects the visual information processing at the newly eye-fixated positions. Therefore, the P100 component of EFRP could be a useful index of how closely drivers examined the visual information in a natural driving environment.

In the present study, we examined the variation of drivers' attention with different workloads during simulated driving. The number of saccadic eye-movements and the EFRPs were measured as indices of drivers' attention. The experiment was approved by the National Institute of Advanced Industrial Science and Technology (AIST) Ethics committee.

\section{EXPERIMENT}

\subsection{Purpose}

In the experiment, drivers were required to perform either a verbal working memory task or a spatial working memory task during simulated driving. It has been proposed that spatial working memory is based on a different mechanism than verbal working memory [15]. Thus, it is possible that the verbal working memory task and the spatial working memory task may yield different patterns for the drivers' attention. Indeed, it was reported that ocular behavior during driving varied greatly with the spatial task when compared to the verbal task [7].

\subsection{Method}

Sixteen drivers (14 male and 2 female; 20-46 years of age) who had normal or corrected-to-normal vision participated in this experiment. They were recruited from the employees of the Nissan Motor Co. All participants were licensed drivers who reported driving in their daily life. Fifteen participants had previous experience in a driving simulator.

A high-fidelity driving simulator, manufactured by Mitsubishi Precision Co., was used for the driving task. In this simulator, an actual vehicle (Nissan CIMA 450 VIP, five-passenger sedan with an automatic transmission) was located on a 6 degree-of-freedom electric motion platform, which provided tactile feedback for drivers. The front view was projected on a $180^{\circ}$ screen in front of the vehicle, and the rear view was projected on two screens and one plasma display panel, which were adjusted for each mirror of the vehicle. Auditory stimuli were presented via two speakers located at the front of the vehicle. The physiological signals were measured with a digital bio-amplifier system 5100 (NF Co.).

A three-lane highway was simulated. Participants were required to drive the vehicle in the outside lane (i.e., a cruising lane) at $100 \mathrm{~km} / \mathrm{h}$ without changing lanes. The road was slightly winding, and several vehicles running in the center and the inside lanes overtook the participant's vehicle.

During the driving task, either a verbal working memory task or a spatial working memory task was given. In the verbal working memory task, a randomly generated sequence of alphabet letters (B, D, G, M, N, and Z were excluded from the list, because these letters are difficult for Japanese people to discriminate) was acoustically presented via in-vehicle speakers. In the 4 min experimental session, 160 letters were broadcast with $1.5 \mathrm{sec}$ of stimulus onset asynchrony (SOA). Participants were required to report orally when a target letter was presented. The set of target letters was given at the beginning of each experimental session. There were two conditions varying the magnitude of working memory load. Two letters were the targets in the low load condition, and four letters were the targets in the high load condition. In both conditions, the list of letters contained 32 targets (20\%).

In the spatial working memory task, the Japanese words "migi" (right) or "hidari" (left) were acoustically presented successively, similar to the verbal working memory task. Participants were required to imagine rotating their body $90^{\circ}$ according to the acoustically instructed direction without actual movement. There were two conditions: a low load condition and a high load condition. In the low load condition, a beep sound was presented after two or three instructions of direction were given. When a beep sound was given, participants were required to orally answer the direction of their body in their image by "mae" (front), "migi" (right), "ushiro" (back), or "hidari" (left). In the high load condition, a beep sound was presented after seven or eight instructions were given. The SOA between instructions was $1.5 \mathrm{sec}$, and the SOA between a beep sound and the first instruction of the next trial was $3.75 \mathrm{sec}$. In the 4 min experimental session, 32 trials were tested in the low load condition, and 16 trials were tested in the high load condition. Note that, because the stimuli in the spatial working memory task were auditory presented, it is plausible that the task involved not only the spatial load but also the verbal load. Thus, in the case that both the verbal and spatial working memory tasks influenced drivers' attention, it is difficult to specify the relationship between the load type and drivers' attention.

The 16 participants were divided into two groups: the verbal working memory task group and the spatial working memory task group (8 participants in each group). Each participant completed six experimental sessions, which consisted of two high load sessions, two low load sessions, and two no-task sessions. In the no-task session, participants performed the driving task while ignoring the 
auditory stimuli. At the beginning of each session, the vehicle stopped at the starting position (the outside lane of highway). Then participants accelerated the vehicle to 100 $\mathrm{km} / \mathrm{h}$. After the speed of the vehicle became stable, the auditory task was started. The order of conditions was counterbalanced across participants.

The EEG signal was elicited from the occipt $(\mathrm{Oz})$ referred to linked ear lobes. The ground lead was attached to the middle of the forehead. To estimate the number of saccadic eye-movements and specify the time of each eyefixation, electrooculographic (EOG) signals were also measured. A pair of electrodes was placed at the outer canthi of the eyes for the horizontal EOG, and another pair of electrodes was placed at the infra- and the supraorbital of the right eye for the vertical EOG. The EEG and EOG were amplified with AC differential amplifiers at a low frequency time constant of $3.3 \mathrm{sec}$ and a high frequency cutoff of $100 \mathrm{~Hz}$. The signals were digitized every $5 \mathrm{msec}$ and recorded on a hard disk. Because the temporal frequency of a typical EFRP ranges from $2 \mathrm{~Hz}$ to $5 \mathrm{~Hz}$, the sampling frequency and filter used in the present study are suitable for estimating the amplitude of P100 component. By visual inspection, a person who was blind to the experimental conditions detected eye-fixation pauses from EOG signals. On average, 227.2 fixation pauses were detected in each condition. Note that, because the electrophysiological signals were digitized every 5 msec, small error $( \pm 5 \mathrm{msec})$ could occur in specifying the time of each eye-fixation. To reduce the influence of such errors, a mean amplitude of 50-150 msec was used as an index of the P100 amplitude. It seems that the possible error $( \pm 5 \mathrm{msec}$ ) against the $100 \mathrm{msec}$ averaging epoch is negligible.

\subsection{Results}

The mean error rates for the verbal working memory task were $0.98 \%$ in the low load condition and $2.77 \%$ in the high load condition. A $t$-test yielded a significant difference of error rates between the low load condition and the high load condition $[t(7)=2.49, p<.05]$. The mean error rates for the spatial working memory task were $0.59 \%$ in the low load condition and $13.3 \%$ in the high load condition. A $t$-test yielded a significant difference of error rates between the low load condition and the high load condition $[t(7)=2.90, p<.05]$. These results indicate that for both working memory tasks, the high load condition was more difficult than the low load condition. Direct comparison of the error rates for the verbal working memory task and for the spatial working memory task is inappropriate because the error rates in the verbal working memory task indicate errors of cognition for each auditory stimulus, whereas those in the spatial working memory task indicate errors for each set of stimuli ( 2 or 3 stimuli in the low load condition and 7 or 8 stimuli in the high load condition).

The mean number of saccadic eye-movements for each condition is shown in Table 1 . The number of saccadic eye-movements was subjected to a one-way repeated measure analysis of variance (ANOVA) with workload (no-task, low load, and high load) as the within-subject factor separately for each working memory task. The main effect of load was significant for both the verbal working memory task $[F(2,14)=6.21, p<.05]$ and the spatial working memory task $[F(2,14)=7.11, p<.01]$. The results indicate that the number of saccadic eyemovements decreased with increased workload for both the verbal working memory task and the spatial working memory task.

Although the relationship between the sizes of saccadic eye-movement and the voltage changes in EOG varies with several factors (e.g., electrode positions), the EOG voltage is often used as an index of saccade size if all conditions were measured within a participant. In the present study, each participant performed no-task, low load, and high load conditions within an experimental session. Thus, the effect of workload on the saccade sizes can be examined by the voltage changes in EOG. The mean voltage changes in EOG corresponding to saccadic eye-movements were $113.9 \mu \mathrm{V}, 121.8 \mu \mathrm{V}$, and $118.7 \mu \mathrm{V}$ for the no-task, low load, and high load conditions in the verbal working memory task respectively, where those were $98.1 \mu \mathrm{V}, 104.7 \mu \mathrm{V}$, and $92.1 \mu \mathrm{V}$ for the notask, low load, and high load conditions in the spatial working memory task respectively. One-way repeated measure ANOVAs showed no significant effect of workload for both the verbal $[F(2,14)=0.66, p=.53]$ and spatial $[F(2,14)=1.22, p=.32]$ working memory tasks.

The EEG signals were averaged by time-locking to fixation pauses to obtain EFRPs in each participant. The averaged waves of EFRPs across participants (i.e., grand averaged waves) are shown in Figure 1. The zero of the $\mathrm{x}$-axis indicates the time of the offset of saccadic eyemovements (i.e., at the onset of eye-fixation pauses). As Figure 1 shows, the most prominent positive component

Table 1: The mean number of saccadic eye-movements (times/min) in each condition

\begin{tabular}{cccc}
\hline & No-task & Low & High \\
\hline VERBAL & 30.3 & 26.6 & 24.9 \\
\hline SPATIAL & 35.0 & 31.0 & 22.7 \\
\hline
\end{tabular}

Note: VERBAL indicates the verbal working memory test group, and SPATIAL indicates the spatial working memory test group. 

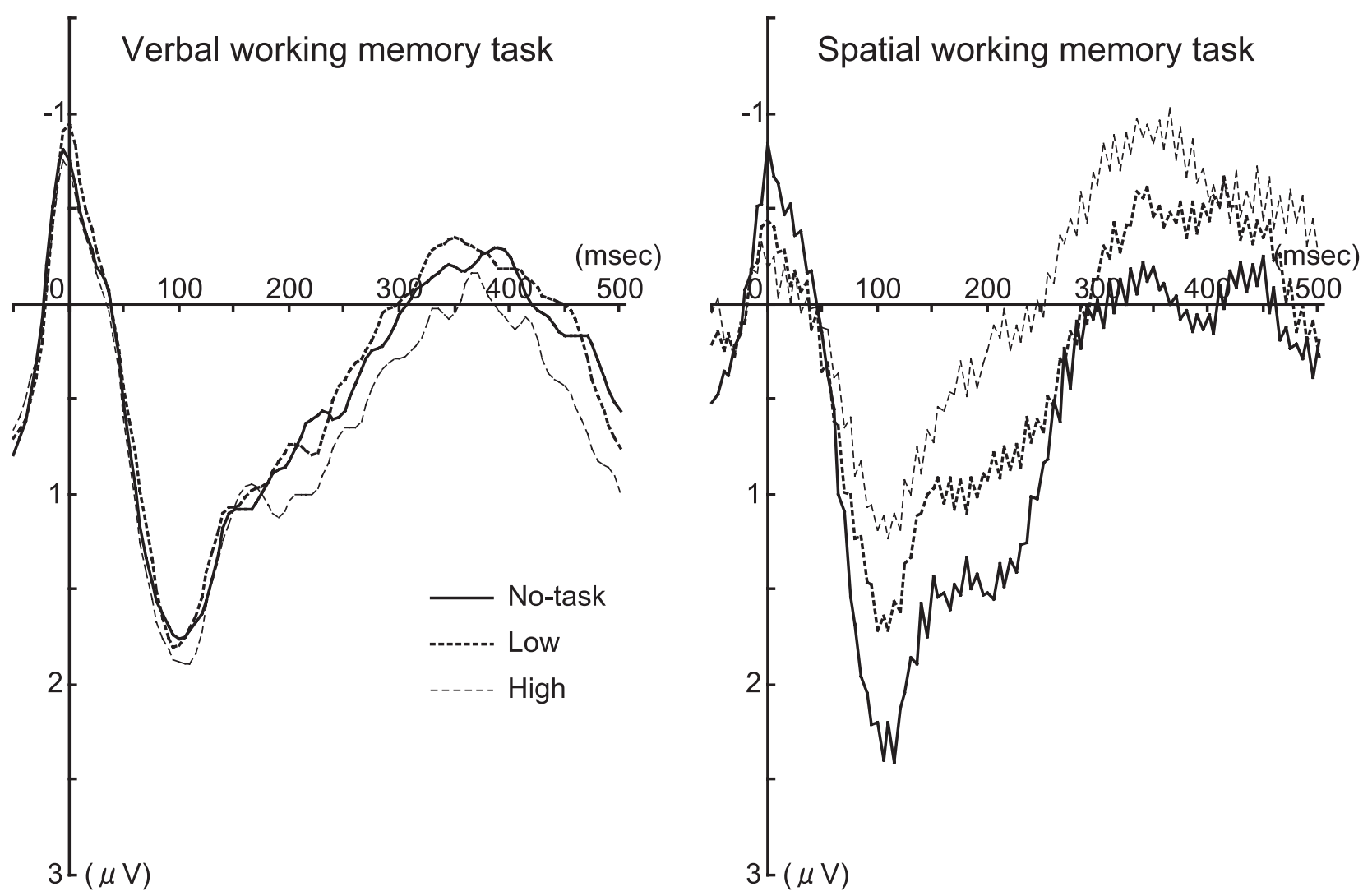

Figure 1: The grand averaged waves of EFRP in the present study. The results from the verbal working memory task group are shown in the left panel, and those from the spatial working memory task group are shown in the right panel.

appeared approximately $100 \mathrm{msec}$ after the offset of saccadic eye-movements in all conditions; this is the P100 component. A mean amplitude of 50-150 msec was computed as an index of the P100 amplitude, where a mean amplitude of a period from $50 \mathrm{msec}$ to $0 \mathrm{msec}$ prior to saccade offset was used as a baseline. The P100 amplitude in each condition is shown in Table 2. The P100 amplitude was subjected to a one-way repeated measure ANOVA with workload (no-task, low load, and high load) as the within-subject factor separately for each working memory task. The main effect of workload was observed in the spatial working memory task $[F(2,14)=4.08$, $p<.05]$, but it was not observed in the verbal working memory task $[F(2,14)=0.20, p=.82]$. These results indicate that the P100 component of EFRP was affected by the spatial working memory load, but not by the verbal working memory load.

Table 2: The amplitude of P100 component of EFRP $(\mu \mathrm{V})$ in each condition

\begin{tabular}{cccc}
\hline & No-task & Low & High \\
\hline VERBAL & 1.38 & 1.31 & 1.47 \\
\hline SPATIAL & 1.64 & 1.16 & 0.80 \\
\hline
\end{tabular}

Note: VERBAL indicates the verbal working memory test group, and SPATIAL indicates the spatial working memory test group.

\section{DISCUSSION}

In the present study, we examined the effects of working memory load during simulated driving by measuring the number of saccadic eye-movements and the P100 amplitude of EFRP. It is reasonable to consider that the number of saccadic eye-movements indicates the frequency of access to visual information, while the P100 amplitude of EFRP indicates how closely drivers examined the visual information. Thus, the results in this experiment suggest that the verbal working memory load reduced the frequency of input of new visual information, but the inputted information was processed accurately regardless of the workload. On the other hand, the spatial working memory load influenced both the frequency of input and the accuracy of visual processing. Therefore, it is possible that the effect of the spatial working memory load during driving is qualitatively different from that of the verbal working memory load.

In the verbal working memory condition, it is plausible that drivers could perform time-sharing processing between driving and the task, which yielded an accurate representation of the visual environment, although the frequency of input was reduced. On the other hand, in the spatial working memory condition, the decline of accuracy of visual processing during driving is inescapable. Based 
on the results of the present study, an in-vehicle device that increases the spatial working memory load of drivers is not as commensurate with safe driving as compared to a device that increases verbal working memory load. However, further research is needed to clarify the generalization of this difference.

As mentioned above, the number of saccadic eyemovements can be related to the frequency of access to visual information, that is, how many locations drivers attend to. On the other hand, the amplitude of the P100 component can be related to the visual processing in each eye-fixated location, that is, how closely drivers examined the visual information. Therefore, these two measures reflect different aspects of visual attention. This account was supported by the results in the experiment, in which an increased spatial working memory load resulted in a decline in both the number of saccadic eye-movements and the P100 amplitude, whereas the verbal working memory load did not affect the P100 component. This dissociation suggests that the number of saccadic eyemovements and the amplitude of the P100 component vary independently and reflect different functions of visual attention. By measuring EFRP, we can examine drivers' attention in more detail, compared to only measuring the saccadic eye-movements. For example, the difference of effects between a verbal task and a spatial task during driving has been reported [7]. However, the mechanism yielding these different effects has not been clarified. For this issue, the present results from EFRP suggest a hypothesis that drivers can perform time-sharing processing between driving and a verbal task. Although further research is needed to establish this hypothesis, there is no doubt that EFRP can be a useful indicator of drivers' attention.

A second important finding of the present study is that EFRP is measurable in a natural driving environment. In the most of the previous studies, reaction times and error rates for drive-irrelevant events (e.g., the onset of LEDs on the windshield or a simulated display) or simulated drive-relevant events (e.g., braking) were used as indices of drivers' attention [4]. Those kinds of measures may interfere with natural driving behavior. In contrast to those indices, EFRP is measurable without any additional stimulus, although placement of several electrodes is needed. This advantage is important for applying EFRP to natural driving environments.

To use EFRP as an index of drivers' attention, we have to consider the temporal resolution and the individual differences of EFRP. EFRP is measurable by averaging EEGs time-locked to the offset of saccadic eye-movements. In general, averaging of more than 50 eye-fixation pauses is necessary to yield a precise wave of EFRP. In the present study, the saccadic eye-movements occurred about 30 times per minute. Thus, the data collected over 2-4 minutes should be averaged to assess the driver's attention with EFRP. This indicates that EFRP is not suitable for assessing short-term variations in a driver's attention.

Individual differences in the pattern of EFRP also limit the usability of EFRP as an index of a driver's attention. EFRP is measured from the human scalp. Thus, the pattern of EFRP varies with the physical properties of each person, such as the form of the brain. Indeed, in the present study, the P100 component of EFRP for one participant was approximately five times as large as that in another participant. To estimate drivers' attention excluding individual differences, it is necessary to measure a baseline of EFRP for each participant, for instance as was done in the no-task conditions in the present study. At the same time, we should not place a strong emphasis on the absolute values of EFRP amplitude because of individual differences.

In conclusion, the present study demonstrated that the P100 component of EFRP could be a useful measure for assessing drivers' visual attention, and that it is measurable in natural driving environments. Although there are some limitations as discussed above, measuring EFRP is helpful for understanding drivers' attention.

\section{ACKNOWLEDGEMENT}

We thank Noriharu Kubo for assistance in collecting the data.

\section{REFERENCES}

1. McKnight, A. J., and McKnight, S.: The effect of cellular phone use upon driver attention. Accident Analysis \& Prevention, 25, (3), pp.259-265 (1993).

2. Rumar, K.: The basic driver error: Late detection. Ergonomics, 33, (10-11), pp.1281-1290 (1990).

3. Strayer, D. L., Drews, F. A., and Johnston, W. A.: Cell phone-induced failures of visual attention during simulated driving. Journal of Experimental Psychology: Applied, 9, (1), pp.23-32 (2003).

4. Patten, C. J. D., Kircher, A., Östlund, J., and Nilsson, L.: Using mobile telephones: Cognitive workload and attention resource allocation. Accident Analysis \& Prevention, 36, (3), pp.341-350 (2004).

5. Matthews, R., Legg, S., and Charlton, S.: The effect of cell phone type on drivers' subjective workload during concurrent driving and conversing. Accident Analysis \& Prevention, 35, (4), pp.451-457 (2003).

6. Backs, R. W., Lenneman, J. K., Weltzel, J. M., and Green, P.: Cardiac measures of driver workload during 
simulated driving with and without visual occlusion. Human Factors, 45, (4), pp.525-538 (2003).

7. Recarte, M. A., and Nunes, L. M.: Effects of verbal and spatial-imagery task on eye fixations while driving. Journal of Experimental Psychology: Applied, 6, (1), pp.31-43 (2000).

8. Recarte, M. A., and Nunes, L. M.: Mental workload while driving: Effects on visual search, discrimination, and decision making. Journal of Experimental Psychology: Applied, 9, (2), pp.119-137 (2003).

9. Isreal, J. B., Chesney, G. L., Wickens, C. D., and Donchin, E.: P300 and tracking difficulty: Evidence for multiple resources in dual-task performance. Psychophysiology, 17, (3), pp.259-273 (1980).

10. Kramer, A. F., Trejo, L. J., and Humphrey, D.: Assessment of mental workload with task-irrelevant auditory probes. Biological Psychology, 40, (1-2), pp.83-100 (1995).

11. Yagi, A.: Saccade size and lambda complex in man. Physiological Psychology, 7, (4), pp.370-376 (1979).

12. Takeda, Y. Sugai, M., and Yagi, A.: Eye fixation related potentials in a proof reading task. International Journal of Psychophysiology, 40, (3), pp.181-186 (2001).

13. Yagi, A.: Visual signal detection and lambda responses. Electroencephalography and Clinical Neurophysiology, 52, (6), pp.604-610 (1981).

14. Kazai, K., and Yagi, A.: Comparison between the lambda response of eye-fixation related potentials and the P100 component of pattern-reversal visual evoked potentials. Cognitive, Affective, \& Behavioral Neuroscience, 3, (1), pp.46-56 (2003).

15. Baddeley, A. D.: Working memory. Oxford: Oxford University Press (1986).

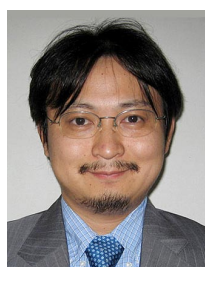

Yuji TAKEDA

Yuji Takeda is a senior research scientist in National Institute of Advanced Industrial Science and Technology (AIST). He received the Ph.D degree in psychology from Kwansei Gakuin University (2001). His research interests include visual attention, memory, and brain function during cognitive tasks.

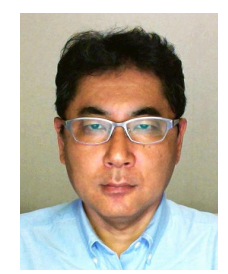

Noritoshi YOSHITSUGU

Noritoshi Yoshitsugu is a manager of vehicle component test group in NISSAN MOTOR Co., Ltd. He has responsible for new vehicle developing ergonomics evaluation point of view.

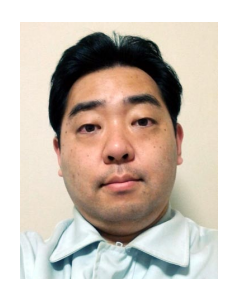

\section{Kazuya ITOH}

Kazuya Itoh is an engineer in Nissan Motor Co., LTD. He has responsible for driver's attention evaluation method and driving simulator experiment technology.

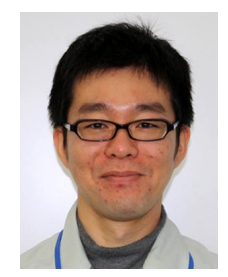

Nobuhiro KANAMORI

Nobuhiro Kanamori currently works as a staff engineer in Panasonic Corporation. He received the Ph.D degree in psychology from Kwansei Gakuin University (2004). His research interests include the evaluation and development of products by the methods of ergonomics and Kansei 